\title{
POLITIQUE ET POÉTIQUE DES FLUX. LA STRATÉGIE DU RÉSEAU DANS LA BANDE DESSINÉE DU COLLECTIF TRANSNATIONAL CHICKS ON COMICS
}

\author{
Marie Lorinquer-Hervé
}

\begin{abstract}
Résumé : Dans le contexte de la mondialisation culturelle des années 2000, les bandes dessinées circulent de façon croissante d'un pays à l'autre. Pour autant, ce passage des frontières ne s'accompagne pas nécessairement d'une ouverture des frontières du champ lui-même, dont certains acteurs demeurent aux marges. En 2008, c'est en réaction à la position périphérique occupée par les femmes en bande dessinée que le collectif Chicks en Comics voit le jour. Les dessinatrices qui le composent se proposent de rompre l'isolement en s'appropriant et en subvertissant les outils de la mondialisation pour mener une discussion transnationale autour des questions de genre sur un blog commun. Mené en bande dessinée à travers le principe du cadavre exquis, cet échange a favorisé le développement d'une véritable poétique des flux.
\end{abstract}

Mots-clés : Chicks on Comics, bande dessinée, mondialisation, flux, écriture en collaboration, femmes, marges

\section{POLITICS AND POETICS OF FLOWS. THE NETWORK STRATEGY IN THE TRANSNATIONAL COLLECTIVE CHICKS ON COMICS}

\begin{abstract}
In the context of the cultural globalization of the 2000s, comic strips increasingly circulate from one country to another. And yet this everintensifying border-crossing isn't followed by an opening of the borders of the field itself as some of its protagonists are excluded from the process. The Chicks on Comics collective is created in 2008 as a reaction to the marginal position that women hold in the comic strip industry. By making the tools of globalization their own, the female artists seek to put an end to their isolation and to make way for a transnational conversation about gender on a community-owned blog. Conducted through the exquisite corpse technique, this conversation contributed to the development of an authentic poetics of the flows.
\end{abstract}

Keywords: Chicks on Comics, comic strip, globalization, interaction, collaborative writing, women, margins

Dans son ouvrage éponyme, Olivier Dolfus définit la mondialisation comme un "monde de la concentration, de toutes les concentrations " qui " entretient des vides» (DOLFUS, 2007, p. 43). Tandis que les éléments les plus puissants fonctionnent en système, d'autres demeurent isolés aux périphéries 
des nouveaux espaces concentrés. La bande dessinée n'échappe pas à cette logique. L'intensification de l'économie de marché et la globalisation culturelle génèrent, en marge d'une production mainstream, des espaces périphériques.

À propos de l'état de la bande dessinée européenne dans les années 40 et 50, Bart Beaty explique déjà : « (...) the industry as it was established in the postwar period consisted of a relatively small number of specialist publishing houses emphasizing mass-market magazine publishing with an eventual goal of consolidating a back catalogue of bestsellers in book form. " (BEATY, 2007, p. 23). En dépit d'évidents particularismes régionaux, cette analyse dit bien les dynamiques qui se mettent en place à une échelle mondiale, et que l'on peut se risquer à résumer à grands traits. L'impératif de rentabilité qui se renforce avec cette industrialisation de la production impose à la création des cahiers des charges relativement strictes. Pour autant, ces contraintes n'empêchent pas le développement et la publication d'une bande dessinée dite "d'auteurs », soit que les auteur-rice-s s'approprient la contrainte, soient qu'ils s'en émancipent. La seconde option est notamment prise à partir de la fin des années 60. Les velléités libertaires qui parcourent alors le monde imprègnent le champ de la bande dessinée qui s'enrichit d'une production plus expérimentale. Ces renouvellements sont toutefois freinés dans les années 80 . Sous l'effet de la période de récession, les marchés éditoriaux nord-américain et européen se tournent plus fermement vers une " bestseller mentality " (BEATY, 2007, p. 27). La même concentration de la production est observable en Amérique du Sud et notamment en Argentine, où une période de dictatures et d'instabilité politique fait l'effet d'un "golpe a los libros" (GOCIOL, INVERNIZZI, 2010), bientôt aggravé par une crise économique dans les années 90. Progressivement, les nouveaux auteur-rice-s et les travaux novateurs se voient alors poussés dans les marges du marché, si ce n'est en dehors. L'auteur français JeanChristophe Menu, co-fondateur de la maison d'édition indépendante L'Association, souligne cette opacification de la frontière entre un marché mainstream et une « marginalité de fait » :

En 1990, il n'y avait pas de demi-mesure. Soit on était dans l'amateurisme, et en général on se limitait à publier un périodique plus ou moins bien fabriqué, et toujours diffusé confidentiellement (et une part du Fanzinat militant 
POLITIQUE ET POÉTIQUE DES FLUX.

LA STRATÉGIE DU RÉSEAU DANS LA BANDE DESSINÉE DU COLLECTIF TRANSNATIONAL

CHICKS ON COMICS

tenait, et tient toujours, à cette marginalité de fait) ; soit on était dans la sphère de l'Édition avec ses contingences lourdes (même les éditeurs de taille moyenne qui nous [L'Association] avaient précédés et qui étaient nos modèles comme Futuropolis, Artefact ou Le Square s'étaient retrouvés dans des engrenages de distribution industrielle qui les avaient d'ailleurs condamnés, ces structures ayant toutes été absorbées, respectivement par Gallimard, Veyrier et Albin Michel). (MENU, 2011, p. 190)

La précision que le dessinateur apporte entre parenthèses est importante. Les années 90 sont fréquemment présentées comme une période féconde pour la bande dessinée, animée par des franges indépendantes qui introduisent, contre la bande dessinée mainstream, de nouveaux formats et de nouveaux genres (au premier rang desquels le roman graphique et l'autobiographie). Il faudrait cependant souligner le fait que cette indépendance, avant d'être réappropriée et revendiquée, était une situation "de fait ». La concentration du champ de la bande dessinée avait créé et désigné ses autres.

En outre, loin de reposer sur de seuls critères formels, cette altérisation a été, et est encore, renforcée par d'autres marqueurs cumulables. PowerPaola, autrice de bande dessinée équatorio-colombienne installée en Argentine, rappelle à propos de ses débuts dans la première moitié des années 2000 : «Cuando yo empecé a hacer cómics, había que escuchar cierta música, vestirse de cierta forma, ser hombrey dibujar de cierta manera. Entonces, no entraba por ningún lado (...) » (je souligne) (CHICKS ON COMICS, 2016). À l'échelle mondiale, la bande dessinée s'est en effet constituée comme un " territoire masculin » (ACEVEDO, 2016). Ces dernières années, les polémiques déclenchées par les listes de nommé.e-s exclusivement ou essentiellement masculines pour le Grand prix d'Angoulême en France (2016) et le Troféu HQMIX au Brésil (2017) ont encore mis en lumière l'invibilisation des femmes en bande dessinée et les "barrières invisibles" qui leur sont opposées (ACEVEDO, 2010, p. 55-67). En dépit du succès retentissant de quelques autrices comme Marjane Satrapi en France, Alison Bechdel aux États-Unis, Julie Doucet au Canada, ou Maitena en Argentine, ces mécanismes d'exclusion se sont en 
outre reproduits dans la bande dessinée dite " alternative », indépendante des grandes maisons d'édition, très majoritairement masculine.

C'est ce constat d'une alternative qui manque d'alternatives que dressent les dessinateurices ${ }^{1}$ PowerPaola et Anna Bas Backer (Allemagne) lorsqu'iels se rencontrent en Espagne en 2008. À ce moment, iels se perçoivent réciproquement comme "la otra que hacía historieta " (CHICKS ON COMICS, 2016). La marginalité que dénote cette perception est d'autant plus saisissante que les deux dessinateurices vivent et travaillent sur deux continents différents. Par désir d'amoindrir ce sentiment d'isolement d'abord, et d'ouvrir un dialogue avec des pairs ensuite, iels se mettent au défi de lancer un blog collectif en faisant appel à quelques autrices dont iels connaissent l'existence. PowerPaola convoque ainsi les Argentines Delius, Caro Chinaski, Clara Lagos et Sole Otero, tandis qu'Anna Bas Backer fait appel à deux sœurs allemandes, Lilli et Ülla Löge, ainsi qu'à Maartje Schalkx, dessinatrice hollandaise installée en Angleterre. En septembre 2008, les dessinateurices lancent ensemble le blog "Chicks on Comics », hébergé par la plateforme en ligne gratuite Blogspot. Au fil des années, le blog passera à la plateforme Tumblr et verra le départ des sœurs Löge (2014), de Sole Otero (2017) et de Maartje Schalkx (2018), ainsi que l'arrivée de la Néerlandaise installée à Londres Julia Homersham (2011-2016), de la Brésilienne Fabiane « Chiquinha » Langona (2015-2016), de la Singapourienne Pixin Weng (2017-) et de la Lettonne Zane Zlemesa (2017-).

Le format du blog collectif n'est pas nouveau. De fait, quatre des membres du groupe (PowerPaola, Caro Chinaski, Clara Lagos et Sole Otero) officient déjà sur le blog "Historietas Reales" au moment du lancement de "Chicks on Comics ». Lancé en 2005, le blog argentin fonctionne sur le mode anthologique, réunissant un groupe fluctuant de dessinateur.rice-s qui postent tour à tour une ou plusieurs planches autobiographiques au fil des jours de la semaine. Dans un contexte éditorial défavorable, la dimension collective de

\footnotetext{
${ }^{1}$ Dans un souci d'inclusivité, les membres du collectif Chicks on Comics seront ci-après désignés par le pluriel "dessinateurices», associé au pronom neutre «iels». De la même manière, on parlera de « fxmmes ». Cette terminologie a vocation, d'une part, à éviter l'écueil d'un binarisme qui ne saurait rendre compte du fait que la subalternité est ici formée par opposition à la catégorie bien particulière des hommes cisgenres. D’autre part, elle permet de prendre en compte la transition de genre opérée par Joris Bas Backer, annoncée en 2016. Le nom « Anna Bas Backer » sera exclusivement conservé dans les cas où il sera question d'un moment ou d'une production antérieurs à 2016.
} 
POLITIQUE ET POÉTIOUE DES FLUX.

LA STRATÉGIE DU RÉSEAU DANS LA BANDE DESSINÉE DU COLLECTIF TRANSNATIONAL

CHICKS ON COMICS

cette plateforme autogérée présente entre autres avantages celui de décupler la visibilité de chacun·e des auteur.rice-s. Leurs lectorats respectifs confluent sur un même blog et peuvent ainsi, d'une part, découvrir le travail d'autres artistes (vers les blogs individuels desquels sont proposés des liens) et, d'autre part, accéder aux dernières publications de leurs auteur.rice-s de prédilection sans avoir à consulter plusieurs blogs (DE LUCA ; DELORENZI, 2009). « Chicks on Comics " reprend à son compte cette pratique du regroupement, que l'on pourrait qualifier de stratégie nodale de visibilité, en y ajoutant une dimension intersectionnelle. La visibilité offerte par le blog profite en effet à différents degrés aux membres du collectif selon qu'iels jouissent en amont d'un capital symbolique plus ou moins important au sein du champ, mais également selon leur ancrage dans des pays du Nord ou du Sud (si les bandes dessinées européennes et nord-américaines sont traduites et diffusées dans les pays du Sud, l'inverse est moins vrai), selon l'état du marché de la bande dessinée dans la région de laquelle iels sont issu·e.s, et selon leur identité de genre (si les femmes cisgenres sont peu représentées en bande dessinée, les personnes queer ou transgenres le sont bien moins encore). En somme, "Chicks on Comics » intègre dans un même réseau informel des éléments issus des marges plus ou moins lointaines du champ de la bande dessinée et de la mondialisation économico-culturelle de manière générale.

La formation de ce réseau informel se fait au moyen de la réappropriation de certains outils de la mondialisation. Le collectif reprend à son compte les pratiques participant du fonctionnement d'un réseau culturel mondial exclusif et en fait le support d'un discours alternatif. Il configure en ce sens un underground qui épouse le "nouveau contexte d'une culture mondialisée » (GROENSTEEN, 2009). Les dessinateurices font notamment un usage consommé des NTIC (Nouvelles Technologies d'Information et de Communication) pour mettre en place des flux souterrains. Gratuites et faciles d'utilisation, les plateformes de publication en ligne sont envisagées comme aptes à fonctionner comme des nœuds transnationaux. Les échanges s'y font au travers des publications, mais également en amont, en coulisse, à travers des e-mails et des services de messagerie instantanée, et en aval, dans la section commentaires des publications. La communication est facilitée par l'emploi du globish, anglais rudimentaire caractéristique de la globalisation que le collectif 
élit comme langue véhiculaire. Il permet à la fois de dépasser les frontières langagières entre les membres et d'atteindre un lectorat plus large. L'élargissement de ce lectorat est par ailleurs favorisé par la facilité de diffusion qu'offre l'auto-publication en ligne. À la différence du fanzine papier, distribué localement et dans un nombre d'exemplaires limité, le blog présente l'avantage d'être diffusable de manière instantanée, gratuite et illimitée dans le temps (DELORENZI, DE LUCA, 2009). L'apparition des hashtags, qui accompagnent systématiquement les publications à partir du passage au Tumblr en décembre 2015, témoigne d'ailleurs d'un usage conscient de ces potentialités d'expansion.

Par ailleurs, ce discours est conçu comme alternatif non seulement à la concentration masculine dans le champ globalisé de la bande dessinée, mais également à l'androcentrisme qui en découle. Le collectif Chicks on Comics se donne à la fois pour objectif d'employer la bande dessinée comme un outil de communication et de décloisonnement, et de mettre ces échanges à profit pour mener une réflexion collégiale sur le genre. Dans une vignette autoréflexive postée sur le blog, Delius affirme ainsi: "We are united by comics and by reflecting on genre, which is necessary to understand who we are " (je souligne) $(T, 57)^{2}$. De fait, ce projet est mis en application dès la vignette inaugurale du blog en forme de question manuscrite posée par PowerPaola : " How to do a "girls only" comics blog? " (B, 1). L'interrogation n'est pas sans rappeler celle de la dessinatrice argentine Patricia Breccia, une vingtaine d'années plus tôt. Dans les premières cases de la bande dessinée "El plumín, ¿ovula o no ovula? » publiée en 1987 dans un numéro spécial de Fierro dédié aux femmes, la dessinatrice se représentait assise à sa table à dessin, se demandant sur le mode de l'ironie: “¿Qué tenía que hacer yo? ¡Ah...! Sí... Una historieta para una sección de mujeres... dirigida por hombres " (ACEVEDO, 2010, p. 76-78). Le caractère autogéré de " Chicks on Comics » ne déplace que peu la question. Le blog n'est pas, comme l'était la revue Fierro, dirigé par des hommes, mais le choix des termes " girls only ", placés entre guillemets, dit la persistance des attentes qui pèsent sur la mise en place d'un tel projet. Un blog tenu par des fxmmes et, a fortiori, thématisant cette présence, a tôt fait d'être perçu et

${ }^{2}$ Les références des dessins publiés sur "Chicks on Comics » à travers les plateformes Blogspot (B) et Tumblr ( $T$ ) seront ci-après indiqués sous la forme (B ou $T$, numéro de la publication). 
POLITIQUE ET POÉTIOUE DES FLUX.

LA STRATÉGIE DU RÉSEAU DANS LA BANDE DESSINÉE DU COLLECTIF TRANSNATIONAL

CHICKS ON COMICS

désigné comme une autre bande dessinée. C'est-à-dire, non comme une bande dessinée produite par des fxmmes et sur les fxmmes, mais defemmes et pour les femmes. Cette essentialisation est, de fait, régulièrement observable dans les médias à travers l'emploi du qualificatif " girly " (et de ses équivalents dans d'autres langues : " de minas », " das minas », « féminine », etc.) pour désigner cette bande dessinée, comme le rappelle le Collectif des créatrices de bande dessinée contre le sexisme dans sa charte (BDEGALITE, 2013).

La récupération de ce terme dans la question posée fonctionne à la fois comme une mise en accusation et comme un retournement du stigmate. Le même procédé est répété dans le nom même qu'a choisi de se donner le collectif. Le mot d'argot "chicks" est une autre façon de désigner péjorativement les femmes. La valeur militante de la réappropriation du terme est ici d'autant plus claire que le nom "Chicks on Comics" est aussi une référence et un hommage aux Chicks on Speed, groupe de musique électronique féministe allemand de la fin des années 90. Le clin d'œil rend manifeste le positionnement politique du collectif et l'inscrit dans une histoire des fxmmes dans la production culturelle, à laquelle les dessins postés au cours des onze années suivantes n'auront de cesse de rendre hommage à travers la mention plus ou moins explicite de fxmmes artistes. Ce désir de visibilisation de la place des fxmmes dans le domaine culturel est en outre souligné en creux dans le passage de "Chicks on Speed" à "Chicks on Comics". Le remplacement de la drogue (speed) par la bande dessinée (comics) sous-entend une addiction des fxmmes du collectif à la bande dessinée, et donc une affirmation résolue de leur existence dans le champ. En ce sens, la mobilisation de ce vocabulaire esquisse déjà un début de réponse à la question liminaire de PowerPaola. Le blog se donnera comme horizon thématique le questionnement et le dépassement des frontières imposées par les stéréotypes de genre. Le premier dessin qui suit la question posée par PowerPaola en guise de réponse l'illustre de façon exemplaire. Anna Bas Backer y écrit : « These days, when I wasn't out shopping for belts or inside drawing comics, I was reading and rereading Phoebe Gloeckner. The story lines are great and the drawing are kick- 
ass! Everything is autobiographical, schizophrenic, nasty, sexy, dirty, good drawings, good drawings, good drawings. » $(\mathrm{B}, 1)$.

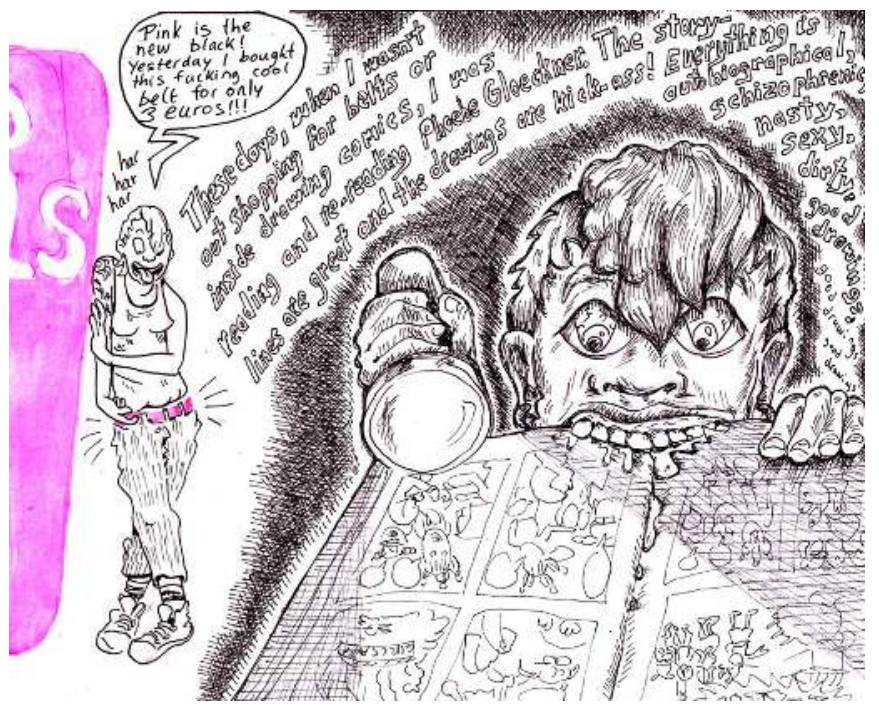

La mention de Phoebe Gloeckner, figure majeure de la bande dessinée underground étatsunienne des années 70 réalisée par des fxmmes (Wimmen's Comix; Twisted Sisters) ancre le projet du collectif dans une généalogie qui atteste de l'existence historique des fxmmes dans la bande dessinée. Les qualificatifs choisis pour décrire son travail sont aux antipodes de la douceur et de la naïveté convoyées par le terme " girls". La possibilité d'une assignation de son travail à des thématiques genrées est balayée par l'insistance sur les qualités de la narration et du trait. Comme un écho à cette ascendance revendiquée, Anna Bas Backer choisit, de plus, de se représenter dans une tenue punk, les yeux écarquillés, en train de mordre et de baver, dans un trait énervé et contrasté. Bien qu'indirecte, la réponse est claire : le « "girls only" comics blog » ne proposera pas une bande dessinée féminine, mais une bande dessinée sur le féminin, en tant que construction sociale.

Ce premier échange entre PowerPaola et Anna Bas Backer met également en marche ce qui sera le mode de fonctionnement du blog. À la façon d'un cadavre exquis, chaque publication est une réponse à la précédente. La réflexion sur les questions de genre est donc menée sur le mode de la 
POLITIQUE ET POÉTIQUE DES FLUX.

LA STRATÉGIE DU RÉSEAU DANS LA BANDE DESSINÉE DU COLLECTIF TRANSNATIONAL

CHICKS ON COMICS

conversation, ouvrant la possibilité d'un dialogue entre des perspectives plurielles. À titre d'exemple, Caro Chinaski propose en décembre 2008 de réfléchir à la question de l'épilation $(B, 8)$. Dans les publications suivantes, le thème est successivement discuté par les différent.e.s auteurices. Ülla Löge évoque les mises en garde qu'on lui a adressées (B, 9), Delius rebondit en évoquant la pénibilité de la pratique $(\mathrm{B}, 10)$, Sole Otero rétorque qu'elle trouve au contraire des propriétés apaisantes à l'épilation (B, 11), et ainsi de suite, jusqu'à ce que Maartje Schalkx conclue la discussion en dessinant une vulve poilue en caméra subjective accompagnée d'une bulle indiquant « Sorry guys, but I really don't care whether you shave or not » $(\mathrm{B}, 15)$, avant que la discussion ne dévie sur les injonctions liées au dating hétérosexuel. La diversité des points de vue exprimés est parfois augmentée par l'invitation ponctuelle de dessinateurices extérieur.e.s au collectif, à l'occasion d'expositions ou d'événements particuliers ${ }^{3}$. Ce dispositif favorise une version collégiale de ce que Hillary Chute nomme une éthique du témoignage et de l'autoreprésentation (CHUTE, 2010). En effet, dans Graphic Women, Life Narrative and Contemporary Comics, la critique souligne le fait que les récits à la première personne menés par des fxmmes en bande dessinée présentent la vertu de rendre à proprement parler visibles des identités et des expériences traditionnellement invisibilisées. Cette visibilité, le dialogue collégial mené par Chicks on Comics la reporte sur la diversité même des points de vue exprimés en lieu et place de l'image uniformisée et stéréotypée de la femme traditionnellement représentée dans l'histoire de la bande dessinée (ACEVEDO, 2009) et des arts visuels de manière générale (NEAUD, 1992).

Pour autant, il faut préciser que, si tel est le projet annoncé par le collectif, le succès de sa mise en application n'est pas toujours évident. En dépit de l'affirmation selon laquelle « uno de los discursos que une al grupo es la ironía constante sobre cuestiones de género » (je souligne), la réflexion promise sur des questions de genre est fréquemment interrompue par des digressions vers d'autres sujets (de l'écologie à la mondialisation, en passant par la gentrification ou la dimension capitaliste des fêtes de Noël). Par ailleurs, les

\footnotetext{
${ }^{3}$ Aux mois de mars et avril 2013, pas moins de 36 invité.e.s (qualifié.e.s de « Guest-Chicks » et « GuestCocks ») répondent ainsi au sujet imposé « Spring \& Feminism » (B, 199-235) à l'occasion d'une exposition des travaux du collectif à Berlin.
} 
discussions menées sont davantage descriptives et anecdotiques que véritablement analytiques - en atteste l'exemple susmentionné de la nature des réflexions proposées pour la thématique de l'épilation. Leurs limites sont parfois mêmes explicitement confessées par les auteurices. Après la mention de la polémique suscitée par le Grand prix d'Angoulême de 2016, Anna Bas Backer explique ainsi : "So much goes on in the world that makes me think. But I don't necessarily have an opinion ready. Also, I'm not sure I should. » (T, 6). Les conversations se heurtent en outre aux incompréhensions et désaccords que peuvent occasionner les perceptions différentielles de certains sujets de société en fonction de l'ancrage socio-culturel des auteurices qui les commentent. C'est notamment ce qui se passe dans les discussions menées entre les mois d'août et octobre 2011 autour du phénomène de la gentrification, auquel Delius et Clara Lagos opposent depuis Buenos Aires des réalités qu'elles jugent plus urgentes (B, 147 : « I'm an unemployed middle-class woman with no time for theory »). Enfin, des difficultés de communication sont régulièrement occasionnées par le choix de l'anglais. La langue n'étant pas maîtrisée au même degré par tous les membres du groupe, elle donne fréquemment lieu à des tournures fautives, voire difficiles à comprendre et raturées. Lorsque Sole Otero évoque en août 2012 (B, 184) une version soustitrée en espagnol du court-métrage documentaire Ilha das Flores (1989), du brésilien Jorge Furtado, l'impossibilité pour une partie des membres du collectif de comprendre un film en espagnol ou en portugais est ainsi rappelée par Ülla Löge dans un anglais lui-même chaotique, dans le fond comme dans la forme $(\mathrm{B}, 185)$.

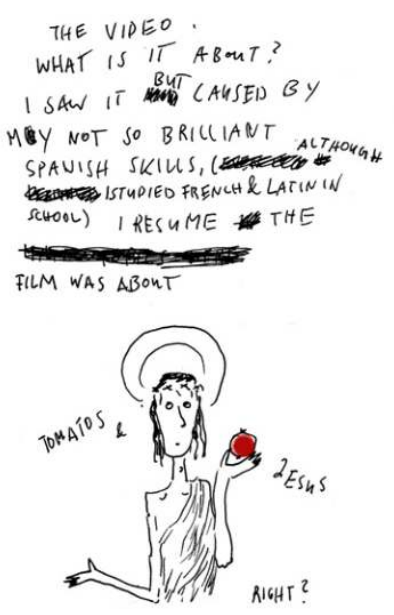


POLITIOUE ET POÉTIOUE DES FLUX.

LA STRATÉGIE DU RÉSEAU DANS LA BANDE DESSINÉE DU COLLECTIF TRANSNATIONAL CHICKS ON COMICS

Les outils et modes de communication choisis par le collectif se révèlent donc, par certains aspects, limitants.

Toutefois, ce qui demeure en dernière instance constamment mis en avant, c'est la dynamique même de l'échange. Polyphonique, symphonique ou cacophonique, il est l'arrière plan permanent du projet et est régulièrement souligné par des mises en abyme. Lorsque Maartje Schalkx exprime son malaise face à la discussion que mènent ses collègues autour de la gentrification (B, 143), elle le fait ainsi en redessinant les posts précédents in situ, sur l'écran d'un ordinateur. De la même manière, après que Joris Bas Backer a évoqué les mobilisations du 8 mars 2017 (T, 37), PowerPaola met en scène la réponse qu'elle lui adresse à travers une autoreprésentation sur l'écran d'un téléphone tenu par son collègue, devant la table à laquelle il s'était luimême représenté dans son dessin ( $T, 38)$. Cette mise en abyme de l'échange est prolongée par une insistance explicite sur la nécessité de la sororité : « As Caro and Clara said, we need each other. We need to be together. " (je souligne).
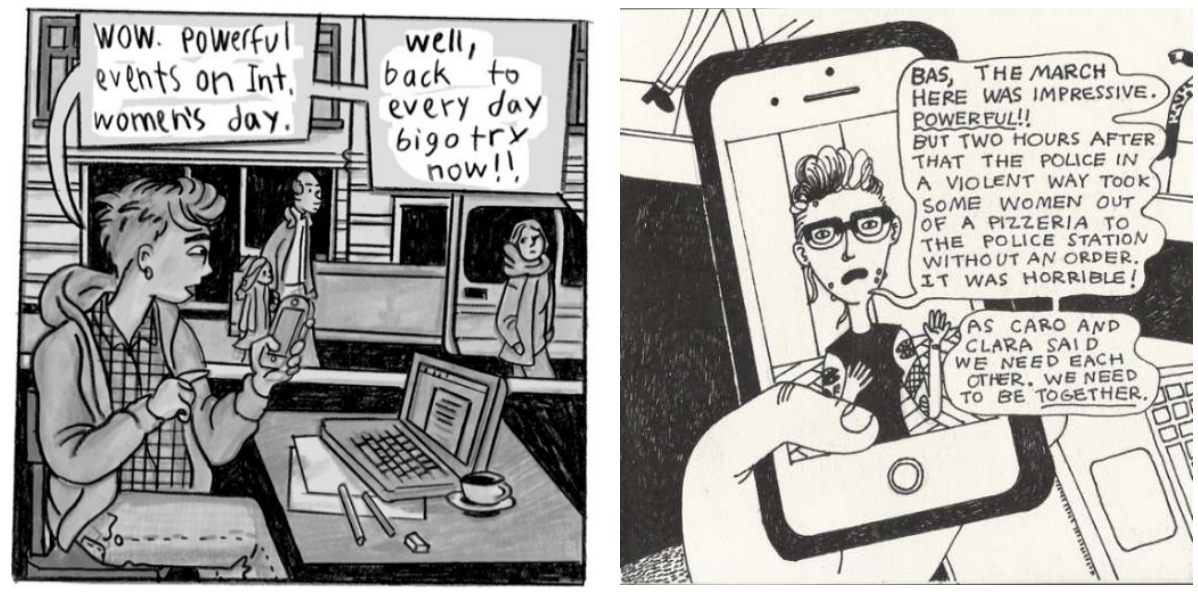

Si des traces de difficultés communicationnelles affleurent souvent dans les échanges du collectif, c'est donc en tant que mise en scène d'un effort volontaire qu'elles sont conservées. Elles mettent en évidence à la fois les mécanismes exclusifs de la globalisation culturelle qui cantonnent certain.e.s acteur.rice-s à l'incommunicabilité, et les liens lancés par le collectif pour dépasser cette situation de fait. C'est ce qu'illustre astucieusement le dispositif choisi pour la première exposition de Chicks on Comics en 2010, à l'occasion du 
festival Viñetas Sueltas (Buenos Aires). Les portraits dessinés de chaque membre du collectif, qui surplombent les dessins exposés, sont reliés entre eux par des fils tendus qui s'entrecroisent en une véritable toile au dessus des spectateurs.

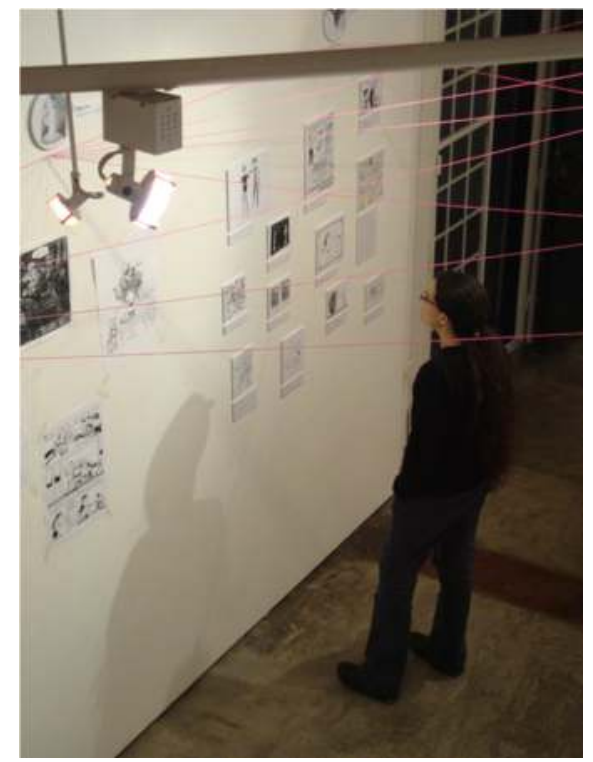

Le réseau ainsi matérialisé se mue en un toit, en un abri apte à recevoir d'autres bandes dessinées. Ce qui se joue dans ces mises en abyme, c'est en somme ce que Bourdieu nomme l' « alchimie mystérieuse » qui permet de passer d'un " espace de relations » contingent, généré par un ensemble de conditions objectives, à un groupe "mobilisé », c'est-à-dire conscient de son existence et de l'action qu'il mène (BOURDIEU, 1984).

En ce sens, le blog ne recouvre pas tout à fait l'une ou l'autre des fonctions de monstration ou de publication que Ricardo De Luca et Florencia Pereyra Delorenzi identifient comme les deux stratégies adoptées par les blogs dans les années 2000 (DE LUCA; DELORENZI, 2009). Puisque "Chicks on Comics " repose sur une interactivité des dessins successivement postés, il n'a pas vocation à être une plateforme de publication d'œuvres complètes des auteurices - qui tiennent de fait, pour la plupart, un blog personnel en parallèle. Et il n'a pas exactement une vocation de monstration, c'est-à-dire de vitrine qui permettrait aux auteurices d'espérer intégrer le marché de la bande 
POLITIOUE ET POÉTIOUE DES FLUX.

LA STRATÉGIE DU RÉSEAU DANS LA BANDE DESSINÉE DU COLLECTIF TRANSNATIONAL

CHICKS ON COMICS

dessinée par le biais de publications au format livre. L'horizon du travail mené par le collectif a davantage à voir avec une extension de ce marché. Il s'agit de questionner l'état de concentration du champ et d'étendre les possibles de la bande dessinée. C'est notamment ce que donnent à penser deux espaces vides apparus sur le Tumblr en 2017 (T. 45, 54). Cette année-là, deux dessins du collectif ont été censurés par la plateforme, qui a agi en autorité éditoriale, rappelant le caractère incomplet de l'autogestion en ligne lorsqu'elle passe par des hébergeurs. Les dessins ont été remplacés par des messages automatiques de justification de la suppression pour cause de " contenu adulte ». Là où les auteurices auraient pu prendre la décision de supprimer les posts concernés afin de ne pas laisser en ligne ces espaces vides (ou, plus précisément, vidés) qui produisent une rupture visuelle dans l'esthétique du blog, iels ont fait le choix de conserver la trace de cette censure. Deux ans plus tard, les messages automatiques sont en effet toujours visibles. Ils agissent visuellement comme des espaces encore à conquérir pour la bande dessinée. Ce que met en place le blog " Chicks on Comics ", c'est donc à la fois une politique des flux, c'est-à-dire un dépassement de la situation d'isolement produite par la mondialisation par le biais de conversations performatives sur le sujet, et une poétique des flux, une ouverture des possibles de la bande dessinée au moyen de cette subversion.

Le mouvement de dépassement des barrières est en effet non seulement mis en scène mais envisagé comme pouvant faire émerger des récits alternatifs aux modèles éculés. Le globish maladroit se mue ainsi en support de création d'une langue mixte. À la manière des littératures bilingues qui explorent des langues frontières (DE COURTIVRON, 2003), les dessins publiés sur le blog jouent régulièrement avec les rencontres linguistiques occasionnées par les échanges internationaux. C'est ce qui se passe, à titre d'exemple, dans la dernière entrevue d'une série imaginée par les auteurices du collectif en 2014. Fidèle au principe suivi dans les sept publications précédentes, Anna Bas Backer interview une artiste extérieure au collectif et restitue graphiquement un entretien dans lequel les lecteur.rice-s n'apprendront pas grand-chose de l'interviewée. Outre qu'une partie du portrait passe, dans une entrevue dessinée, par le trait lui-même, ces interviews dans lesquelles il ne se dit rien ou presque mettent l'accent sur l'échange en lui-même. Maki Shimizu, ici interviewée, est une dessinatrice japonaise installée en Allemagne et 
manifestement peu à l'aise avec l'anglais. Alors que l'on pourrait s'attendre à ce que l'entrevue se passe en allemand et soit restituée en anglais sur blog, Anna Bas Backer choisit mettre en scène les difficultés de Maki Shimizu à parler anglais à travers un jeu de code-switching et de language mixing. Sous l'effet de l'injonction ("In english please. »), le texte et le contour des bulles attribuées à la dessinatrice japonaise deviennent tremblants, ratatinés et raturés. Ses réponses passent sans cesse de l'allemand à un anglais maladroit, rendant l'entrevue difficile à lire.

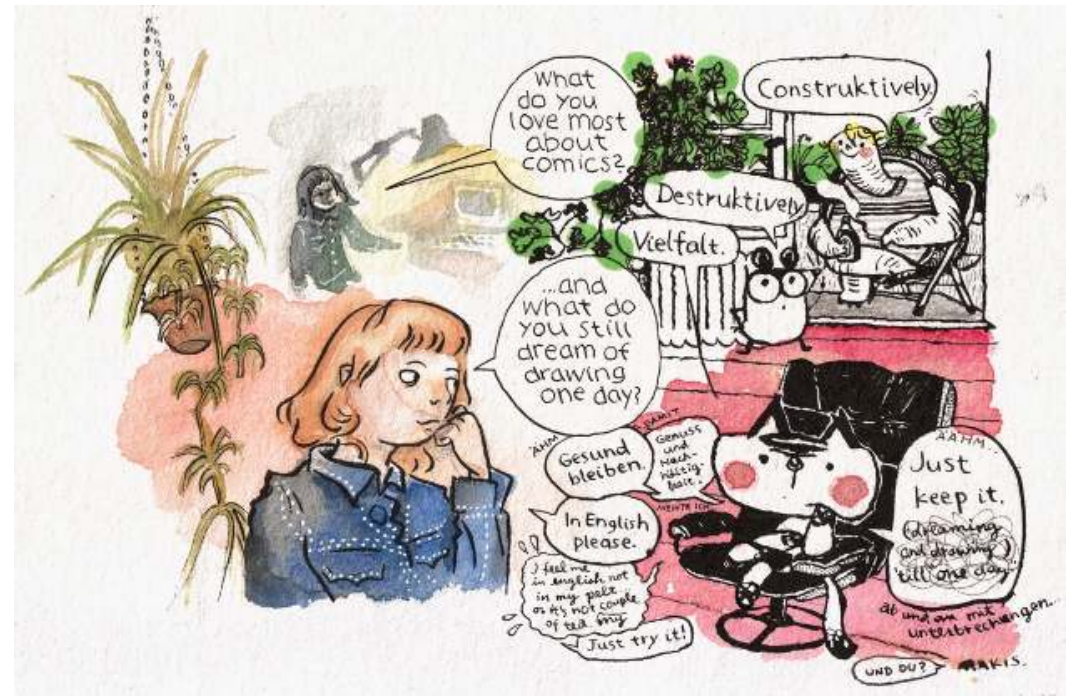

Sur le blog, les parties en allemand sont traduites en anglais, mais la traduction ne les rend pas beaucoup plus compréhensibles. La réponse de Shimizu à la question « What do you still dream of drawing one day? » est ainsi traduite par : " Eh stay healthy so that pleasure and sustainability is what I meant (sic.) ». Cette opacité reporte l'attention des lecteurices sur la pluralité linguistique elle-même et sur l'opération de destruction-construction du langage qui s'y joue - comme le suggèrent les bulles attribuées à des petits personnages à l'arrière plan, dans lesquels on peut lire dans un anglais hybridé d'une graphie allemande : " construktively », " destruktively ». À cet égard, la réponse de Shimizu à la première question qui lui est posée (« What do you love most about comics ? ") est à la fois synthétique et programmatique : "Vielfalt » (la diversité). Contre une globalisation exclusive de la bande dessinée, Chicks 
POLITIQUE ET POÉTIOUE DES FLUX.

LA STRATÉGIE DU RÉSEAU DANS LA BANDE DESSINÉE DU COLLECTIF TRANSNATIONAL

CHICKS ON COMICS

on Comics fait valoir des récits éclatés à l'intérieur desquels les voix se refusent à fusionner.

Le cadavre exquis, mode d'écriture prédominant sur le blog, est l'illustration paradigmatique de ce postulat des vertus créatives du divers. Si le collectif n'est pas le premier groupe d'artistes à adapter en bande dessinée ce jeu des surréalistes - les dessinateurs de l'OuBaPo s'étaient déjà approprié cette contrainte (GROENSTEEN, 2004) - il en fait moins un outil d'expérimentation formelle qu'un mode de décloisonnement de la création. En rendant possibles des continuations imprévues, il écarte la possibilité de définir une fois pour toutes ce que pourra être la bande dessinée qui se crée. De fait, cette invention au fil du pinceau, le collectif en fait son identité même. Sa nature échappe aux définitions fermées. Sur le blog et en dehors du blog, la pratique des auteurices subit des variations et hybridations constantes. De nombreux formats sont explorés, de l'exposition au livre collectif en passant par un Fanzine a la carta (2016) que les lecteur.rice-s ont pu composer et assembler selon leurs désirs à partir d'un éventail de planches de quelques 151 artistes. Des projets plus courts ont ponctuellement vu le jour, à la manière d'inserts, tels que la série d'interviews de 2014 ou de la fiction muette Silent Conversation / Suburbanimal(B, octobre 2014 - octobre 2015). Le collectif a évolué au gré des départs et des arrivées de ses membres (le départ de sœurs Löge et de Julia Homersham, et l'arrivée de Chiquinha, Pixin et Zane Zlemesa a notamment déplacé la dualité marquée en l'Argentine et l'Europe occidentale qui existait au départ), mais aussi de la transition de genre de Joris Bas Backer, annoncée en 2016 (T, 29), qui a conduit le groupe à se redéfinir comme un collectif d'acteurices minorée.es dans le champ de la bande dessinée, davantage que comme un collectif de femmes. Sur le blog, PowerPaola assume et revendique cette plasticité dans un strip autoréflexif $(T, 51)$. Tandis que s'opère un travelling avant menant significativement de la figure de l'artiste à des gros plans confinant à l'abstraction, la dessinatrice insiste sur le fait que Chicks on Comics est d'abord "un collectif», c'est-à-dire, un ensemble de communicantes « indéfini » qui évolue " au gré des circonstances ». 


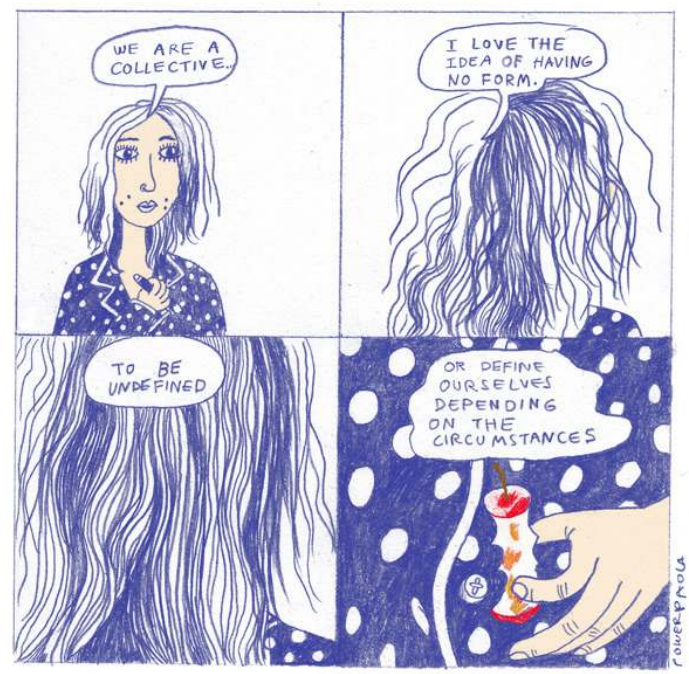

La fluidité que le collectif se donne pour mot d'ordre va en définitive dans le sens de ce qu'Édouard Glissant nomme, contre la mondialisation, une mondialité(GLISSANT, 1997). C'est-à-dire, une mise en contact qui ne se fait pas sur le mode du nivellement et de la concentration (métissage), mais de l'expansion imprévisible au travers de la mise en contact du divers (créolisation).

Au fil des publications et des projets menés, l'échange transnational mis en place par Chicks on Comics s'est en somme constitué comme un récit choral fonctionnant sur le double mode de la conversation, au sens de dialogue, et de la con-versation, au sens d'écriture collaborative. Initialement, le collectif s'était donné pour projet d'utiliser la bande dessinée comme outil de communication pour mener des discussions collectives autour des questions de genre. Mais un retournement s'est progressivement opéré. Cette discussion a permis de questionner, en creux, les potentialités de la bande dessinée. Elle a été - et est toujours - le laboratoire de la possibilité d'une écriture de soi collaborative en bande dessinée. C'est-à-dire, une écriture de soi en tant qu'appartenant à un groupe donné (ici déterminé sur le critère du genre dans le contexte de la mondialisation), et réalisée en collaboration avec d'autres membres de ce groupe. Cette écriture n'est donc ni une autobiographie individuelle, que les auteurices mettent à distance par la moquerie dès la première publication du blog (« neurotic autobiographical shit » $(\mathrm{B}, 1)$ ), ni une 
POLITIQUE ET POÉTIOUE DES FLUX.

LA STRATÉGIE DU RÉSEAU DANS LA BANDE DESSINÉE DU COLLECTIF TRANSNATIONAL

CHICKS ON COMICS

autoethnographie, dès lors que le groupe n'est pas perçu au prisme d'un seul individu, ni un manifeste, puisque les voix ne fusionnent pas. Elle est, bien plutôt, une écriture du réseau, qui fait des flux mondiaux son catalyseur, son objet et sa poétique.

\section{Bibliographie}

ACEVEDO, Mariela et al. (2016). Historieta feminista en América Latina: autoras de Argentina, Chile, Brasil y México. In: Revista Tebeosfera. [En ligne] $<$ https://www.tebeosfera.com/documentos/historieta_feminista_en_americ a_latina_autoras_de_argentina_chile_brasil_y_mexico.html $>$ [consulté le 22 août 2019]

ACEVEDO, Mariela (2010). Imago fémina: ensayo sobre fábulas de heterodesignación y textos de resistencia en las historietas. Buenos Aires: Universidad de Buenos Aires.

ACEVEDO, Mariela (2009). Revista Clítoris. Historietas y exploraciones varias. Feminismo y textualidades. In: Revista Tebeosfera. [En ligne] $<$ https://www.tebeosfera.com/documentos/revista_clitoris._historietas_y_e xploraciones_varias._feminismos_y_textualidades.html> [consulté le 22 août 2019]

BDEGALITE, (2013). Charte des créatrices de bande dessinée contre le sexisme. In: Collectif des créatrices de bande dessinée contre le sexisme. [En ligne] $<$ http://bdegalite.org/> [consulté le 22 août 2019]

BEATY, Bart (2007). Unpopular Culture: Transforming the European Comic Book in the 1990s. Toronto : University of Toronto Press.

BOURDIEU, Pierre (1984). Espace social et genèse des "classes". In : Actes de la recherche en sciences sociales, vol. 52-53 (p. 3-14). Paris : Seuil.

CHICKS ON COMICS, (2017). Un yo colectivo y mundial. [En ligne] $<$ https://youtu.be/UjkGo7CDbf4> [consulté le 22 août 2019]

CHUTE, Hillary (2010). Graphic Women. Life Narrative and Contemporary Comics. New York : Colombia University Press.

DE COURTIVRON, Isabelle (dir.) (2003). Lives in Translation. Bilingual Writers on Identity and Creativity. New York : Palgrave Macmillan.

DE LUCA, Ricardo : DELORENZI Florencia Pereyra (2009). Un abordaje de la historieta argentina desde el ARS. Buenos Aires : Universidad de Buenos Aires. 
DOLFUS, Olivier (2007). La mondialisation. Paris : Presses de Sciences Po.

GLISSANT, Édouard (1997). Poétique IV. Traité du Tout-Monde. Paris: Gallimard.

GOCIOL, Judith ; INVERNIZZI, Hernán (2010). Un golpe a los libros. Buenos Aires : Eudeba.

GROENSTEEN, Thierry (2009). Pour une internationalisation des études sur la bande dessinée dans le contexte de la mondialisation. In : Éditions de l'An 2. [En ligne] <https://www.editionsdelan2.com/groensteen/spip.php?articlel3> [consulté le 22 août 2019]

GROENSTEEN, Thierry (2004). Ce que l'OuBaPo nous révèle de la bande dessinée. In : Neuvième Art 2.o. [En ligne] $<$ http://neuviemeart.citebd.org/spip.php?article547> [consulté le 22 août 2019]

HEIN, Fabien (2012). Do it yourself! Autodétermination et culture punk. Paris : Le Passager Clandestin.

MENU, Jean-Christophe (2011). La bande dessinée et son double. Paris: L'Association.

NEAUD, Lynda (1992). The Female Nude. Art, Obsenity and Sexuality. Londres : Routledge.

Recebido em 22 de agosto de 2019.

Aceito em 30 de agosto de 2019. 
POLITIQUE ET POÉTIOUE DES FLUX

LA STRATÉGIE DU RÉSEAU DANS LA BANDE DESSINÉE DU COLLECTIF TRANSNATIONAL

CHICKS ON COMICS

Pontos de

Interrogação 
